\title{
Reflexiones sobre la estrategia de rehabilitación basada en la comunidad (RBC): la experiencia de un programa de RBC en Bolivia
}

\author{
Reflections on community-based rehabilitation strategy (CBR): \\ the experience of a CBR program in Bolivia
}

Urko Díaz-Aristizabal ${ }^{1}$ Silvia Sanz-Victoria ${ }^{2}$ Milton Sahonero-Daza ${ }^{3}$ Sandra Ledesma-Ocampo ${ }^{3}$ Mesías Cachimuel-Vinueza ${ }^{3}$ Marisabel Torrico $^{3}$
${ }^{1}$ Hospital Pius de Valls. Plaça St. Francesc 1. 43800 Valls España. urkodiaz@hotmail.com

${ }^{2}$ Escola Universitària Creu Roja. Centro Adscrito Universitat Autònoma de Barcelona. Terrassa (España)

${ }^{3}$ Programa Rehabilitación Basada en la Comunidad. Fundación Arquidiocesana Padre Ignacio Zalles (RBC-FAPIZ)

\begin{abstract}
Community-Based Rehabilitation $(C B R)$ is a strategy for community development endorsed by the World Health Organization (WHO), the International Labor Office (ILO) and the United Nations Educational, Scientific and Cultural Organization (UNESCO). It is designed to promote rehabilitation, equal opportunity and social inclusion of Disabled Persons (DP) in their home communities by fostering cooperation among disabled individuals, their families, and other concerned social actors, it encourages community leadership and full social participation by DP through multi-sector cooperation. This article explores the historical antecedents and basic features of CBR strategy through an analysis of a directed culture change initiative developed by a foundation in the Cochabamba administrative region of Bolivia. Especially in intercultural environments, certain aspects of the socio-cultural context may determine whether CBR programs succeed or fail.
\end{abstract}

Key words Rehabilitation, Disability, Community, Poverty, Cultural diversity
Resumen La Rehabilitación Basada en la Comunidad (RBC) es una estrategia de desarrollo comunitario avalada por la Organización Mundial de la Salud (OMS), la Organización Internacional del Trabajo (OIT) y la Organización de las Naciones Unidas para la Educación, la Ciencia y la Cultura (UNESCO), que persigue la rehabilitación, la igualdad de oportunidades y la integración social de las Personas con Discapacidad (PD) en sus entornos. Con este objetivo promueve la colaboración entre las PD, sus familias y los diferentes actores de la comunidad involucrados, así como el liderazgo comunitario y la participación de las PD mediante el impulso de la colaboración multisectorial. Este artículo expone los antecedentes históricos y las características fundamentales de la estrategia de RBC a partir de un programa llevado a cabo por una fundación del departamento de Cochabamba (Bolivia), para después incidir en algunos aspectos referentes al contexto sociocultural, que especialmente en situaciones de interculturalidad, pueden determinar que un programa de RBC tenga éxito o esté abocado al fracaso.

Palabras clave Rehabilitación, Discapacidad, Comunidad, Pobreza, Diversidad cultural 


\section{Introducción}

Se estima que en el mundo existen aproximadamente 650 millones de Personas con Discapacidad (PD), que representan un 10\% de la población mundial. El $80 \%$ de estas personas vive en países denominados "en vías de desarrollo", donde existen limitaciones para el acceso a servicios básicos y restricciones para la participación en la vida diaria de su comunidad. Además, se estima que solamente un 2\% de las PD de estos países tienen acceso a servicios de atención sanitaria y rehabilitación ${ }^{1-4}$.

Para dar respuesta a las necesidades de las PD y sus familias en todo el mundo, y en concreto en los países denominados "en vías de desarrollo", se deben elaborar estrategias desde una perspectiva que incluya la reducción de la pobreza, el desarrollo comunitario, la igualdad de oportunidades, la integración social, la atención sanitaria y la rehabilitación. La estrategia de Rehabilitación Basada en la Comunidad (RBC) incluye todos estos aspectos, por lo que se considera una de las estrategias de elección para luchar por los derechos de las PD y cubrir sus necesidades, sobre todo en estos países denominados "en vías de desarrollo". No obstante, dado que tanto la salud como la discapacidad son parte integral del fenómeno social y no variables independientes que se puedan considerar aisladas de su contexto sociocultural, y que los padeceres se expresan casi siempre a través de representaciones y prácticas locales culturales ${ }^{5}$, la implementación de un programa de RBC requiere una profundización en los diferentes aspectos de este contexto, ya que en numerosas ocasiones la falta de atención en este sentido origina que el desarrollo de un programa de RBC acabe en un fracaso rotundo.

En relación a los aspectos sociales es fundamental destacar que la discapacidad y la pobreza se correlacionan significativamente. La falta de acceso a la atención sanitaria, educación, formación y trabajo que provoca la pobreza, propicia un aumento en la probabilidad de presentar una discapacidad. A su vez, la discapacidad contribuye a aumentar la pobreza, ya que la situación de las PD restringe la posibilidad de recibir servicios básicos de atención sanitaria y rehabilitación, así como el acceso a la formación, al trabajo, al ocio y a otras actividades de su comunidad y de la sociedad. Es por ello que la mayoría de las personas discapacitadas viven en la pobreza ${ }^{4,6,7}$. En este sentido hay que señalar que es fundamental incidir en las condiciones políticas y económicas del contexto en el que se ubican las PD, evitando en la medida de lo posible considerar la pobreza como una característica de los sujetos para admitirla como un factor macrosocial específico ${ }^{5}$.

En cuanto a los aspectos culturales relacionados con la discapacidad, entendemos que tanto el proceso salud/enfermedad/atención como las representaciones, prácticas y saberes relacionados con la discapacidad se tratan de fenómenos universales. De esta manera, la respuesta social a las enfermedades y a la discapacidad asegura la producción y reproducción biosocial de cualquier sociedad, siendo una de las áreas sociales donde se observan mayor cantidad de simbolizaciones y representaciones y un área clásica donde se ha ejercido un control social e ideológico a nivel macro y microsocial en casi todas las sociedades ${ }^{5}$. Los aspectos culturales adquieren especial relevancia en contextos caracterizados por la interculturalidad, ya que en estas situaciones los actores implicados articulan mediante lógicas culturales diversas sus representaciones, prácticas y saberes en relación a la discapacidad. La convivencia de diferentes lógicas culturales relacionadas con la salud, la enfermedad y la discapacidad origina una hibridación que se caracteriza por su variabilidad y por las relaciones de hegemonía y subalternidad, de manera que el análisis previo de las características y articulaciones de estas lógicas deviene imprescindible para el desarrollo exitoso de un programa de $\mathrm{RBC}^{5,8-10}$.

Para repasar los antecedentes históricos de la estrategia de RBC hay que remitirse a la Segunda Guerra Mundial, cuando al sobrevivir un gran número de soldados y civiles con alguna discapacidad se desarrolló una nueva especialidad biomédica, la rehabilitación, que paulatinamente fue adquiriendo importancia dentro de la investigación médica y tecnológica. En 1951 se creó la Unidad de Rehabilitación de Naciones Unidas para facilitar la transferencia de dichos avances médicos y tecnológicos a los países denominados "en vías de desarrollo", basándose en el entrenamiento y formación de terapeutas y técnicos en rehabilitación, en el apoyo para la construcción de centros de rehabilitación ubicados en las ciudades y en el desarrollo de un equipo profesional compuesto por diferentes especialistas, como médicos rehabilitadores, psicólogos, fisioterapeutas o terapeutas ocupacionales. A pesar de este esfuerzo internacional, la mayoría de las PD no se beneficiaban de esta ayuda, ya que la mayor parte de ellas vivían en zonas rurales o periurbanas ${ }^{11}$.

En 1978 la Organización Mundial de la Salud (OMS) desarrolló la Declaración de Alma Ata, donde se planteaba un modelo integral y progre- 
sista denominado Atención Primaria de Salud, que trasladaba el apoyo y los servicios de salud a las comunidades, centralizados hasta ese momento en hospitales e instituciones ubicados en las ciudades. En el seno de esa atención primaria de salud se contemplaba la rehabilitación, surgiendo de esta declaración la estrategia de $\mathrm{RBC}^{12}$. Durante los ochenta y noventa del siglo pasado se pusieron en práctica numerosos programas piloto de RBC en países denominados “en vías de desarrollo" de África, Asia y Latinoamérica. Algunos programas siguieron los manuales desarrollados por la $\mathrm{OMS}^{13}$, otros fueron apoyados por la Organización Internacional del Trabajo (OIT), la Organización de las Naciones Unidas para la Educación, la Ciencia y la Cultura (UNESCO) o el Fondo de Naciones Unidas para la Infancia (UNICEF), y otros se desarrollaron a partir de iniciativas de Organizaciones No Gubernamentales $(\mathrm{ONG})^{11}$. Tras la experiencia adquirida en el desarrollo de estos programas, la OIT, la OMS y la UNESCO elaboraron un documento conjunto en 1994 (que posteriormente ratificaría también UNICEF), en el que establecían las bases generales sobre la estrategia de $\mathrm{RBC}^{14}$. El desarrollo de la RBC posterior a este documento acentuó la adquisición de un enfoque que se percibe como biopsicosocial y multisectorial, donde se enfatiza la cooperación y colaboración de los actores relevantes en los recursos, habilidades e iniciativas para comenzar un programa de RBC. En 2003 se llevó a cabo una Reunión Consultiva Internacional en Helsinki con todos los actores implicados para realizar una revisión de la estrategia, donde las conclusiones que se obtuvieron se utilizaron para elaborar el nuevo documento de posición conjunta de la OIT, la OMS y la UNESCO en $2004^{15}$. Desde aquel año se celebran de forma anual encuentros para el desarrollo de la estrategia de RBC bajo el auspicio de la OMS.

\section{Rehabilitación basada en la comunidad \\ Qué es la Rehabilitación Basada en la Comunidad?}

La RBC se define como una estrategia dentro del desarrollo general de la comunidad para la rehabilitación, igualdad de oportunidades e inclusión social de todos/as los/as niños/as y adultos con discapacidad, que se aplica a través de los esfuerzos combinados de las personas con discapacidad, sus familias y las comunidades, y los apropiados servicios de salud, educación, formación vocacional y servicios sociales ${ }^{14,15}$.

Dentro del ámbito biomédico de la rehabilitación, se pueden reconocer tres modelos de intervención que no son necesariamente excluyentes entre sí, sino que pueden ser complementarios. Cada uno de ellos tiene sus ventajas e inconvenientes.

En el modelo tradicional o institucional, las PD acuden a un centro de rehabilitación, bien de forma ambulatoria, bien en carácter de internamiento, donde reciben los cuidados y tratamientos específicos que requieren (técnicas de fisioterapia, educación especial, actividades de la vida diaria, etc.). Su gran fortaleza reside en la concentración de recursos humanos y técnicos especializados y sus debilidades son la dificultad para acceder a estos servicios en muchos países y el alto coste que supone su mantenimiento y su sostenibilidad, además del riesgo de segregación de las PD (Figura 1).

Por otro lado, en el modelo de rehabilitación por extensión, un equipo de especialistas de un centro de rehabilitación se desplaza hasta un área concreta, donde realizan una valoración y un plan de intervención de las PD, sin trabajar con el resto de la comunidad. Este modelo tiene la ventaja de acceder a lugares remotos para atender a PD

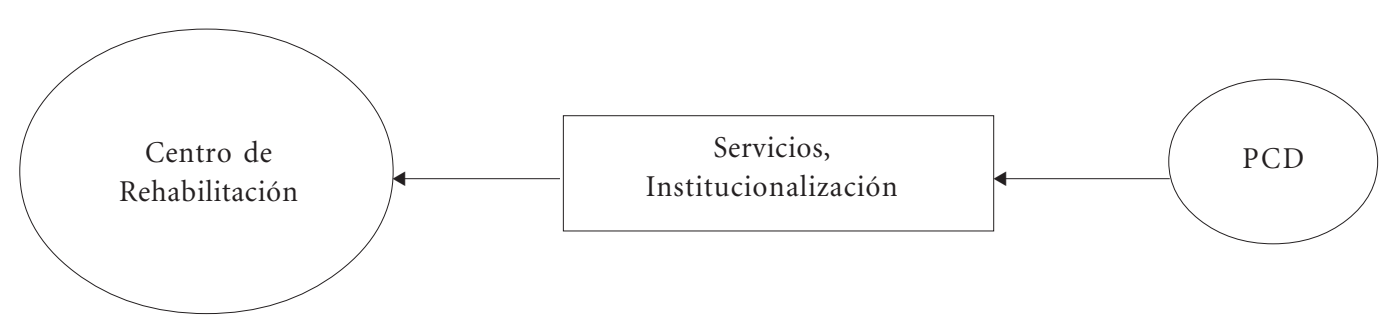

Figura 1. Modelo de rehabilitación tradicional o institucional. 
que de otro modo no tendrían acceso a estos servicios. Por otro lado, presenta los inconvenientes de la sostenibilidad debido a las dificultades de coordinación con todos los especialistas del equipo y la dificultad de viajar de forma regular, por lo que el seguimiento de las PD no resulta satisfactorio. Además, se trata de un servicio que generalmente no parte de una necesidad percibida por la comunidad, por lo que no se traduce en un cambio de actitudes de la comunidad frente a sus miembros con discapacidad (Figura 2).

La estrategia de RBC, por último, no se considera como un modelo en sí, ya que no existe una única manera de desarrollar el programa, sino que son muchas las formas posibles de realizarlo, de acuerdo a las condiciones específicas de la comunidad con la que se trabaja. En esta estrategia se realiza una atención integral de las PD de una comunidad determinada a partir de un centro de rehabilitación, involucrando a sus familias, así como a los diferentes sectores de la comunidad implicados, y capacitando a un promotor que ejerce de nexo entre el centro de rehabilitación y la comunidad. A su vez, el centro de rehabilitación realiza un papel de referencia y contrarreferencia para las PD (Figura 3).

Atendiendo a los documentos de posición conjunta de la OIT, la OMS y la UNESCO, los objetivos principales de la RBC son, en primer lugar, asegurar que las $\mathrm{PD}$ puedan sacar el máximo provecho de sus facultades físicas e intelectuales, tener acceso a los servicios y oportunidades ordinarios y ser colaboradoras activas dentro de la comunidad y la sociedad, y por otro lado, impulsar a las comunidades a promover y proteger los derechos de las PD mediante transformaciones en la comunidad, consiguiendo su plena inserción ${ }^{14,15}$. Además de estos objetivos

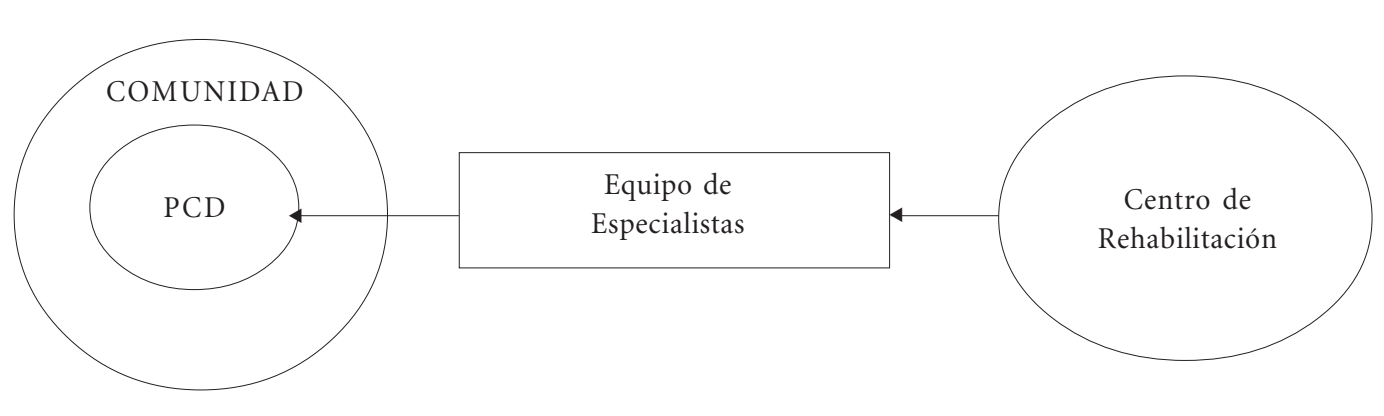

Figura 2. Modelo de rehabilitación por extensión.

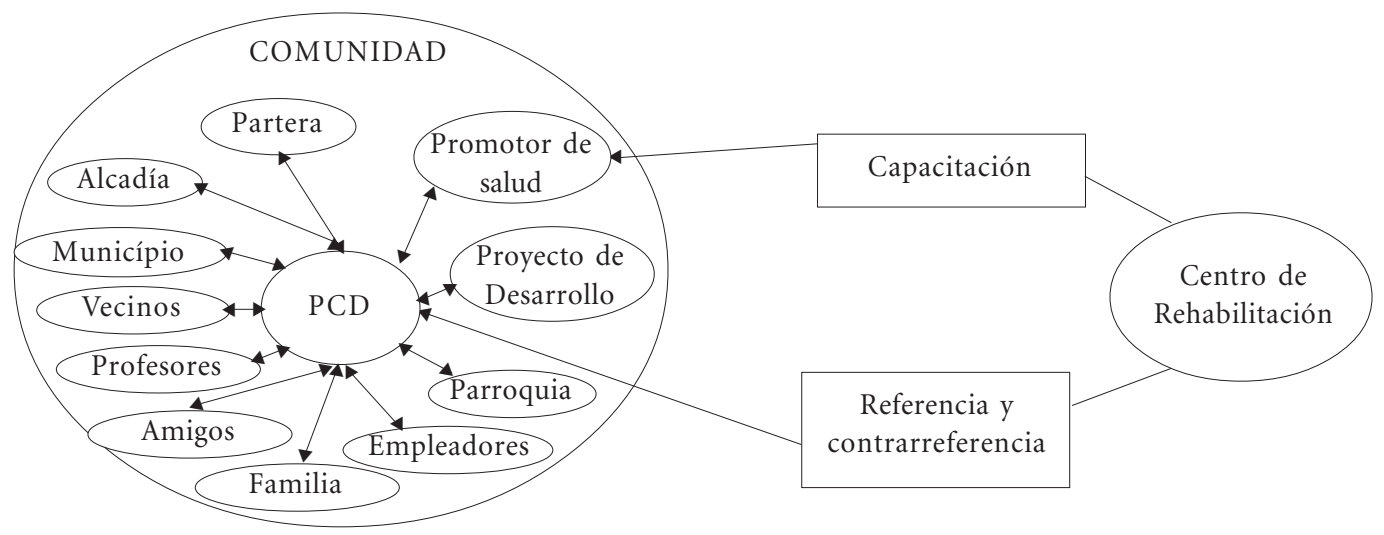

Figura 3. Estrategia de rehabilitación basada en la comunidad. 
fundamentales, un programa de RBC debería incluir objetivos específicos como la creación de una actitud positiva hacia las $\mathrm{PD}$, la provisión de servicios de rehabilitación, la creación de oportunidades para generar ingresos, la facilitación de cuidados de las $\mathrm{PD}$, la provisión de oportunidades de educación y formación, la prevención de causas de discapacidad o la gestión, registro y evaluación del proyecto.

Los programas de RBC se fundamentan en los siguientes aspectos principales:

. Programas basados en la comunidad, incidiendo en la importancia de la participación de la comunidad en la planificación de estrategias, toma de decisiones y puesta en marcha y control del proyecto ${ }^{15,16}$.

- Transferencia de conocimientos, aptitudes y habilidades, capacitando a las PD, a sus familias y a las comunidades con el objetivo de que sean capaces de responder ante los retos y las necesidades que se presenten, no solamente como mera transmisión de conocimientos, sino también de capacidades en la toma de decisiones, resolución de problemas y lucha por sus derechos ${ }^{16,17}$.

- Respeto a las diferencias culturales, suprimiendo el etnocentrismo occidental que presupone la superioridad sobre la población autóctona y teniendo en cuenta las tradiciones locales, el conocimiento tradicional y las estructuras, redes y actividades comunitarias ${ }^{16,17}$. La dificultad estriba en que la RBC surgió de la lógica de especialistas occidentales que no provienen de la cultura donde se asientan los programas.

- Utilización de los recursos locales, tanto materiales como humanos. Se relaciona con la transferencia de conocimientos, aptitudes y capacidades a los implicados y con la sostenibilidad del programa, ya que el objetivo fundamental es que la comunidad dependa lo menos posible de ayudas externas ${ }^{16,17}$.

Los ámbitos de acción de un proyecto de RBC se pueden clasificar en tres niveles. En primer lugar se interviene a nivel primario, donde el objetivo es prevenir la aparición de deficiencias, abarcando una amplia gama de actividades como vacunaciones, atención primaria de salud, protección del medio ambiente o estimulación de la higiene. En segundo lugar se actúa a nivel secundario, que corresponde a intervenciones que reducen o superan las consecuencias discapacitantes y abarca actividades como técnicas de fisioterapia, ayudas compensatorias, actividades de la vida diaria o educación especial. Por último, se interviene a nivel terciario, minimizando las barreras sociales y físicas que excluyen a las PD de la comuni- dad, tales como el fortalecimiento de Organizaciones de Personas con Discapacidad (OPD), la inclusión de mujeres discapacitadas en los grupos de mujeres, el reconocimiento de derechos o la integración escolar de niños con discapacidad. Los programas de RBC más tradicionales se centraban exclusivamente en la acción secundaria, pero pronto quedó patente la necesidad de una intervención en los tres niveles de acción, con lo que el ámbito de actuación se expandía, aumentando así la dificultad de llevar a cabo el programa de forma aislada. Así adquirió importancia el planteamiento de que la discapacidad no es un problema relacionado únicamente con la salud, sino que también está vinculado a múltiples aspectos sociales, incidiendo en la dimensión social de la enfermedad y de la discapacidad ${ }^{15-17}$.

La intervención en los tres niveles de acción y la coordinación con los diferentes sectores implicados conlleva la necesidad de elaborar convenios de cooperación, tanto con las instituciones públicas como con las privadas que trabajen en el área. En los recientes años se ha acentuado este enfoque multisectorial, que enfatiza la cooperación con otros actores relevantes en el desarrollo de la comunidad. De esta manera adquieren importancia en un programa de RBC las PD, sus familias, sus comunidades, los gobiernos (local, regional, nacional), las ONG, las OPD, los profesionales de los diferentes sectores involucrados, las instituciones privadas y los medios de comunicación ${ }^{15-17}$.

Durante el I Encuentro Nacional de Experiencias de $R B C$, celebrado en Cochabamba (Bolivia) en junio de 2007, se estableció la siguiente metodología de desarrollo orientativo de un programa de RBC. En primer lugar se realiza el contacto con las autoridades locales y comunales para explicar el proyecto, tanto con los actores políticos como con los responsables de salud y educación, así como con las diferentes instituciones públicas y privadas que trabajen en la comunidad, con el objetivo de elaborar convenios de cooperación. Se debe desarrollar un marco de actuación, especificando los criterios de selección tanto de las comunidades como de las PD que se incluirán en el programa, considerándose que éste puede fracasar si a priori no se establece dicho marco. Por otro lado, es fundamental que sea la misma comunidad la que solicite la inclusión en el programa de RBC, a través de una PD o de algún representante de dicha comunidad, ya que así se facilita que la comunidad haga suyo el proyecto, siendo mayor su implicación ${ }^{15-17}$. Se continúa con la socialización del proyecto en la comunidad y en los diferentes sectores que la integran 
(madres, OPD, escuelas, postas sanitarias, etc.), además de realizar actividades de concienciación y sensibilización.

La elección del promotor se realiza en base a unos requisitos concretos, como la vocación de servicio, el compromiso, la responsabilidad, el establecimiento en la comunidad, el reconocimiento y aceptación por parte de la comunidad, el saber leer y escribir, ser una PD joven o un familiar de una PD por estar más sensibilizados, o el disponer del apoyo familiar para realizar sus actividades. La función del promotor consiste en identificar a las PD de su comunidad, establecer un vínculo con ellas y sus familias, ayudarles a identificar sus necesidades, formular un plan de rehabilitación, identificar y movilizar los recursos disponibles, hacer referencia a servicios especializados si fuera preciso, asegurar que se cumplen las fases de rehabilitación planificadas y mantener el contacto con la comunidad. Junto con el promotor, se identifica a las PD de la comunidad mediante un censo, realizando una valoración y un informe. Seguidamente se realiza un diagnóstico comunitario para conocer las necesidades de las $\mathrm{PD}$, valorando además la percepción de la comunidad hacia las PD. Se desarrolla un proceso de capacitaciones diferenciadas a los diferentes sectores implicados, con especial énfasis en el promotor, y se procede a la intervención de las PD en diferentes áreas en base a sus necesidades (técnicas de fisioterapia, actividades de la vida diaria, ayudas compensatorias, orientación y movilidad, apoyo escolar, comunicación alternativa, etc.). Simultáneamente se realiza un trabajo conjunto para la integración y la inclusión de las PD en la familia, la escuela y la comunidad. Por último, se lleva a cabo un proceso de monitoreo y seguimiento, tanto de las PD y sus familias, como de los promotores y sus comunidades en general, adaptando la intervención en función de los resultados que se vayan obteniendo.

A pesar de la variabilidad en la forma de aplicar la estrategia de RBC se han identificado algunos factores comunes que pueden afectar a la sostenibilidad del proyecto ${ }^{15-17}$. En primer lugar, es fundamental el reconocimiento de la necesidad del proyecto por parte de las PD y sus familias, además de la disposición de la comunidad a responder a sus necesidades, explorando las diferentes estrategias y recursos existentes. También adquiere relevancia el acceso a servicios de apoyo y recursos, ya que la sostenibilidad del proyecto se incrementa si se coordina con políticas públicas o con programas privados que trabajen en la zona.
Otro aspecto fundamental es la colaboración multisectorial con los diferentes actores, así como el papel de los promotores locales, incidiendo su responsabilidad, su compromiso y su formación directamente en la sostenibilidad del proyecto. Por último, los programas de RBC deben integrarse en los servicios del gobierno, sean a nivel local, regional o nacional, ya que así tienen mayor probabilidad de ser sostenibles.

La estrategia de RBC también presenta algunas debilidades. Primero, se ha enfatizado excesivamente el rol de los profesionales, en su mayoría extranjeros que trabajan en cooperación, con valores, prácticas y concepciones diferentes a los de la cultura donde se trabaja. Otro obstáculo registrado con frecuencia es la dificultad de la colaboración multisectorial, sobre todo en aquellos programas sin integración en políticas nacionales, regionales o locales. La falta de recursos para la capacitación de los promotores comunitarios o para el mantenimiento de los programas es otra de las dificultades, así como el que la comunidad haga suyo el programa. La falta de participación de las OPD, la poca comunicación entre la comunidad y los centros de referencia y la dificultad para incluir en los programas a todos los tipos de discapacidad se perciben, finalmente, como otras debilidades de la estrategia de RBC.

\section{Un programa de $\mathrm{RBC}$}

en el contexto boliviano

La situación de la discapacidad en Bolivia se caracteriza por la situación de exclusión y desigualdad en que se encuentran la mayoría de las $\mathrm{PD}$, siendo víctimas de discriminación en los diferentes procesos de desarrollo económico y social del país. Cotidianamente se vulneran sus derechos fundamentales en su familia y en su entorno social, situación que se agrava por las condiciones de pobreza en las que viven la mayor parte de las PD $(60 \%)^{18,19}$. Por otra parte, es imprescindible destacar el carácter multiétnico del país, donde a pesar de reconocerse desde la anterior Constitución Política del Estado, la mayoría de los programas estatales en salud no incorporan planteamientos interculturales ${ }^{8-10}$.

Bolivia carece de un Registro Nacional de Personas con Discapacidad, por lo que se estima que en la actualidad el 10\% de la población boliviana presenta algún tipo de discapacidad, aproximadamente 800.000 personas (física 3\%, intelectual $3 \%$, sensorial $3.5 \%$, otras $0.5 \%$ ). De acuerdo a las mismas aproximaciones, se estima que menos del 1\% de las PD reciben atención en institu- 
ciones especializadas o servicios de rehabilitación ${ }^{18,19}$. A pesar de la existencia de abundante normativa internacional y nacional referente a la protección y defensa de las $\mathrm{PD}$, su cumplimiento ha sido tradicionalmente muy limitado. Además de la normativa internacional, en Bolivia se promulgó la Ley No 1678 de la Persona con Discapacidad en diciembre de 1995, donde se establecían los derechos, deberes y garantías de las PD, además de constituir el Comité Nacional de la Persona con Discapacidad (CONALPEDIS), cuyo objetivo es la orientación, coordinación, control y asesoramiento de políticas y acciones en beneficio de las personas discapacitadas ${ }^{20}$. La Política Nacional de Prevención de Discapacidades y Rehabilitación en Salud, presentada en febrero de 1996, se elaboró como un "instrumento valioso para la formulación de programas, planes y estrategias en beneficio de las PD", pero su cumplimiento fue excesivamente limitado ${ }^{21}$. Posteriormente se elaboraron diversos decretos en relación a los derechos de las PD, como los Decretos Supremos No 24807 de 1997, No 27477 de 2004 o No 28671 de 2006, así como el Plan Nacional de Igualdad y Equiparación de Oportunidades, elaborado en agosto de 2006, aunque en todos los casos la aplicación fue muy limitada o nula.

La Fundación Arquidiocesana Padre Ignacio Zalles (FAPIZ) es una obra de la iglesia católica de Cochabamba (Bolivia) fundada el 5 de abril de 1991. En sus inicios el proyecto se denominó Centro de Rehabilitación y Educación Especial (CREE) y fue a finales de 1994 cuando lograron una infraestructura propia gracias al apoyo de la institución alemana Christoffel Blindenmission (CBM), logrando ampliar esta infraestructura en 1995 mediante la presentación de un proyecto al programa Telemaratón promovido por un canal de televisión nacional. En el año 2001 la institución pasó a la administración de la iglesia católica y se sustituyó el nombre de CREE por el actual nombre de FAPIZ. Los objetivos fundamentales de la institución son, por un lado, promover el desarrollo integral del niño con ceguera, baja visión o discapacidad múltiple (que incluya la discapacidad visual), y por otro, orientar este desarrollo hacia una integración en la familia y la comunidad hasta alcanzar su rehabilitación profesional y su participación en la comunidad en la medida de sus posibilidades. Estos objetivos se intentan lograr a través de los diferentes programas elaborados por la institución.

El FAPIZ está organizado en diferentes áreas de atención. En primer lugar se encuentra el área pedagógica, donde los niños y jóvenes son prepa- rados e integrados en el sistema de educación regular, recibiendo apoyo escolar individualizado con materiales adaptados. En el área de salud son atendidos mediante diferentes tratamientos como estimulación temprana, técnicas de fisioterapia, orientación y movilidad o estimulación visual. El área de promoción laboral se encarga de la inserción laboral de los jóvenes, a través de la terapia ocupacional, la orientación vocacional o diferentes talleres como panadería o cestería. Por último, se encuentra el área de Rehabilitación Basada en la Comunidad (RBC), donde a diferencia del resto de áreas de la institución, se atiende a niños y jóvenes de las comunidades rurales que presenten cualquier tipo de discapacidad, excepto la discapacidad psiquiátrica, basándose en una metodología que se fundamenta en la estrategia de RBC. El equipo de RBC está constituido por un coordinador, una fisioterapeuta, un educador y rehabilitador visual, una profesora de educación especial, un psicólogo y una trabajadora social, beneficiándose temporalmente de la participación de un médico rehabilitador y de una terapeuta ocupacional. Además cuentan con la colaboración de algunos médicos especialistas como neurólogos, traumatólogos, oftalmólogos u otorrinolaringólogos, así como con la de un ortopeda.

El FAPIZ se encuentra ubicado en la ciudad de Cochabamba (provincia Cercado), capital del departamento del mismo nombre, desarrollándose el programa de RBC en 7 de las 16 provincias que constituyen el departamento: Cercado, Quillacollo, Chapare, Arque, Esteban Arce, Germán Jordán y Carrasco. En esta última se trabaja en dos municipios diferentes, Totora y Entre Ríos, por lo que a pesar de pertenecer a la misma provincia se consideran zonas de trabajo independientes (Figura 4). La intervención en las diferentes provincias se caracteriza por la variabilidad en función de los factores contextuales de cada provincia. Las PD atendidas en la provincia Germán Jordán, por ejemplo, están disminuyendo paulatinamente, ya que otra institución situada en una provincia cercana comienza a hacerse cargo de ellas, con lo que probablemente el FAPIZ dejará de trabajar en esta provincia. Por otro lado, la penetración en el Municipio de Tacopaya (Arque) abarca la casi totalidad de las comunidades, ya que se ha logrado establecer convenios con la Alcaldía de Tacopaya y con otras instituciones que trabajan en la zona, involucrando a actores tanto de la administración pública como del sector privado. La distribución de las PD atendidas durante el 2007 así como el número de promotores se pueden observar en la Tabla 


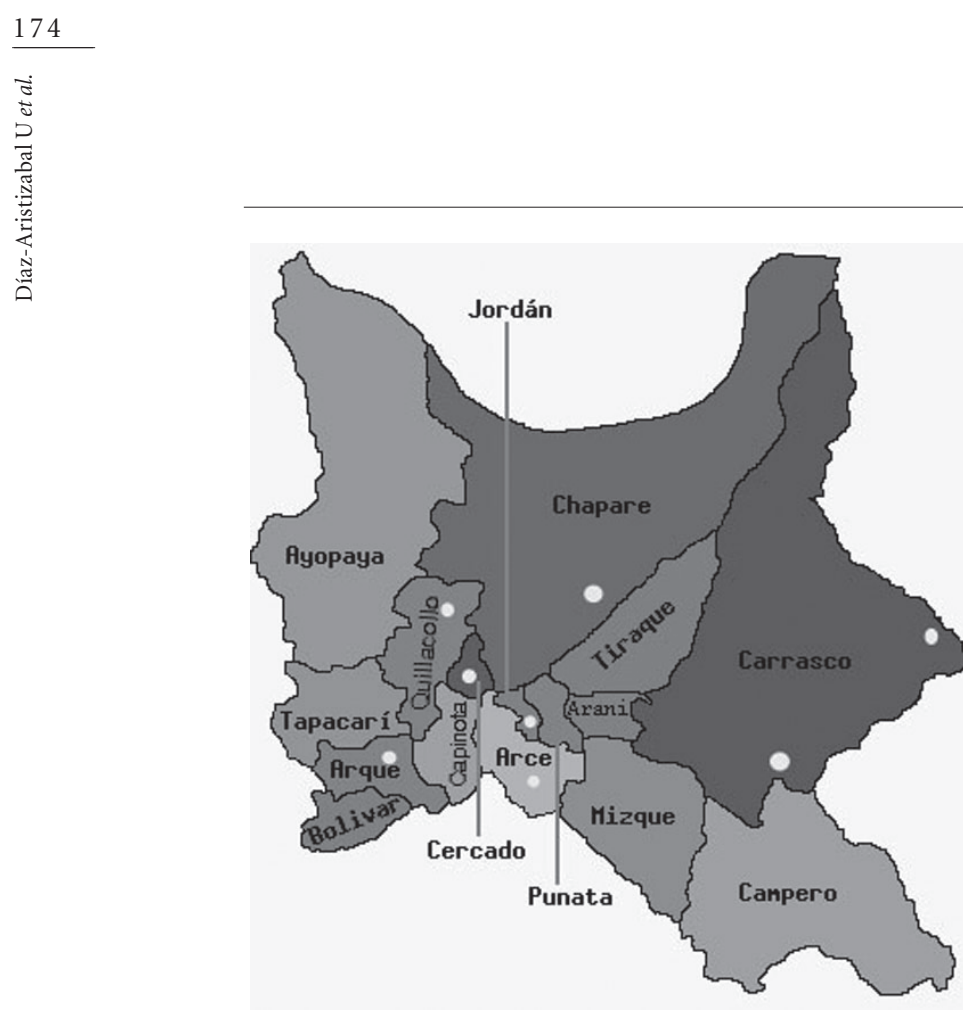

Figura 4. Actuación del FAPIZ en las provincias del departamento de Cochabamba.

1, organizada según áreas geográficas y tipo de discapacidad.

Las actividades que el FAPIZ planifica en las diferentes áreas de actuación se estructuran en seis componentes de abordaje comunitario: concienciación y sensibilización, capacitación, educación y rehabilitación, integración e inclusión, monitoreo y seguimiento, y cooperación y colaboración. En primer lugar, a través de la concienciación y la sensibilización, el programa contribuye a fomentar la buena disposición de los diferentes actores de la comunidad hacia las PD a través de actividades como encuentros periódicos de familias de PD, ferias informativas en escuelas y comunidades, o talleres de concienciación dirigidos tanto a actores locales en el área de salud y educación, como a representantes de otras instituciones que trabajen en la zona. Por otro lado se realizan capacitaciones a los diferentes colectivos involucrados en el programa. El equipo de RBC se capacita mediante la realización de talleres y reuniones periódicas organizados por las instituciones de Cochabamba que trabajan con PD, mientras que de forma mensual se celebra un taller dirigido a la capacitación de los promotores, a los que también se capacita de forma individualizada con las PD de las que son responsables. A través de reuniones periódicas y visitas domiciliarias se instruye a los familiares en el cuidado, manejo y tratamiento de las
PD. Por último, los profesores de niños y jóvenes integrados en escuelas regulares son instruidos en diferentes métodos de enseñanza, como el Braille, el ábaco, métodos de comunicación alternativa para sordos o métodos específicos para el retraso intelectual. En cuanto al componente de educación y rehabilitación, el FAPIZ ofrece tratamiento médico especializado a través de un análisis socioeconómico para las PD que forman parte del programa, además de diferentes tratamientos como técnicas de fisioterapia, actividades de la vida diaria, educación especial o estimulación sensorial. Teniendo como objetivo la integración e inclusión de las $\mathrm{PD}$ en la familia, la escuela y la comunidad en general, tanto las PD como sus familias reciben orientación y apoyo psicológico. El FAPIZ promueve la orientación, preparación y colocación laboral de las $\mathrm{PD}$, además de fortalecer su participación en las escuelas regulares y fomentar su inclusión en la comunidad mediante el diálogo entre las PD y el resto de la comunidad. En el aspecto del monitoreo y seguimiento se interviene tanto en el ámbito de las PD y sus familias, como en el de los profesores y promotores, a través de la planificación, ejecución y evaluación de programas individualizados, realizando visitas domiciliarias semanales o mensuales. Por último, se plantea el mantenimiento y fortalecimiento de los convenios de colaboración establecidos con la administración pública o con entidades privadas, además de buscar el apoyo y la implicación de otras entidades.

\section{Valoraciones finales}

Como hemos observado a lo largo del artículo, la RBC se trata de una estrategia avalada por la OMS, la OIT y la UNESCO que es adecuada para dar respuesta a las necesidades que demandan las PD y sus familias, sobre todo en los países denominados "en vías de desarrollo". No obstante, como también hemos señalado, la estrategia de RBC presenta una serie de dificultades e inconvenientes, entre los que destaca la sostenibilidad del programa. En este sentido, la participación de la comunidad en la planificación y desarrollo del programa es un factor fundamental, ya que nada de lo impuesto u ofrecido como regalo se mantendrá si carece de significado o si no da sentido de pertenencia a la persona que se atiende. La participación solamente se obtiene si se crean las motivaciones adecuadas para que la población sienta la necesidad de intervenir ante un problema social por ella percibido, además 
de requerir la comprensión de los métodos que se utilizan, tanto a nivel individual como colecti$\mathrm{vo}^{22}$. Los dirigentes comunitarios tienen gran influencia en las decisiones finales que adopta la comunidad, por lo que su abordaje deviene un factor fundamental para la sostenibilidad del programa, así como la inclusión en el programa de promotores procedentes de la propia comunidad. Dentro de los requisitos que se establecieron en el I Encuentro Nacional de Experiencias de $R B C$ adquiere importancia que el promotor posea prestigio e influencia en su comunidad.

Con el objetivo de mantener la participación de la comunidad, además, es imprescindible que el programa de RBC se ajuste a unas normas básicas. En primer lugar, es necesario llevar las herramientas que ofrece la biomedicina a las comunidades sin esperar que éstas acudan en su búsqueda, de manera que se requiere movilidad e iniciativa por parte del personal, además de establecer un plan rotativo de visitas que cubra un área extensa de actuación con promotores en las comunidades que consoliden el trabajo. En segundo lugar es fundamental colocar la biomedicina al nivel de la cultura de la comunidad, ya que el objetivo final de un programa de RBC es lograr la generalización de una actitud en la comunidad que favorezca la inclusión y la rehabilitación de las PD, y no la mera imposición de la lógica de la biomedicina. Por último, es importante ajustar la biomedicina a las condiciones del medio social, de manera que el éxito de un programa de RBC no se mide por la perfección que puedan tener sus servicios, sino por el ajuste que se consiga al contexto sociocultural, medido por la aceptación que le conceda la comunidad. Es en este aspecto donde los programas de RBC pueden ser deficitarios, ya que resulta difícil integrar la biomedicina al contexto intercultural considerando la hegemonía que mantiene ${ }^{22}$.

En el ámbito de la antropología se admite que tanto la salud como la discapacidad son aspectos integrados en la estructura social y no variables independientes de su contexto sociocultural. Las enfermedades y las discapacidades se expresan mediante representaciones, prácticas y saberes incluidas culturalmente a niveles locales ${ }^{5}$, de manera que la hibridación de las diferentes representaciones, prácticas y saberes que resulta de la convivencia de distintas culturas producirá unas lógicas nuevas que diferirán en mayor o menor grado de las que han sido originadas, además de articularse entre todas ellas en relaciones de hegemonía y subalternidad. La estrategia de RBC no debe pretender introducir la lógica de la biomedi- cina en el seno de las comunidades a través de relaciones de hegemonía respecto a las demás lógicas, sino que debe tenerlas en cuenta y conocer las características y articulaciones entre dichas lógicas si se quiere asegurar la buena marcha de un programa de $\mathrm{RBC}^{5,8-10}$. Como ocurre en multitud de países, también en Bolivia el sistema médico tradicional está subordinado técnica e ideológicamente a la biomedicina, de manera que generalmente no se tiene en cuenta las representaciones, prácticas y saberes en relación a la enfermedad y a la discapacidad que pueden existir en las comunidades donde se quiere implementar el programa $^{8-10,22,23}$. Por tanto, en grupos de población de cultura heterogénea se debe tomar en consideración no solamente los aspectos sanitarios, sino también los factores sociales y culturales, lo que impone al personal del programa la necesidad de conocer profundamente la cultura de las comunidades donde opera. En este sentido el conocimiento de la lengua nativa y de la lengua nacional deviene en requisito imprescindible, como medio para el correcto entendimiento y para la profundización en el sistema de creencias, valores, prácticas y actitudes de su cultura, sobre todo en relación al ámbito sanitario ${ }^{22}$.

A pesar de las dificultades anteriormente observadas, una de las ventajas que la estrategia de RBC presenta es su flexibilidad, que permite adaptaciones para su aplicación en contextos socioculturales diferentes, especialmente en aquellos que se caracterizan por la interculturalidad. El conocimiento de las características y las articulaciones entre los diferentes saberes y representaciones relativos a la discapacidad es indispensable para la sostenibilidad de un programa de RBC, por lo que previamente a la implementación de un programa de RBC se propone desde este artículo el desarrollo de una investigación en relación a las diferentes representaciones, prácticas y saberes relacionados con la discapacidad que estén presentes en un grupo social específico. Dicha investigación se fundamentaría en la perspectiva planteada por Menéndez desde el punto de vista del actor ${ }^{5}$, ya utilizada en algunas investigaciones localizadas en Bolivia ${ }^{8-10}$, donde el análisis se fundamenta en la información obtenida a partir de todos los actores implicados en una problemática específica, y no solamente en alguno de ellos. Este énfasis en la articulación de las lógicas relacionadas con la discapacidad en el ámbito microsocial, no obstante, debe articularse a su vez con el aspecto macrosocial, de manera que, tal como señala Menéndez, los aspectos políticos y económicos específicos del grupo social 
resultan fundamentales para entender correctamente el ámbito microsocial. De esta manera, la condición de pobreza que se correlaciona directamente con la discapacidad no se trataría de una característica propia del individuo o del grupo social, sino que traduciría unas condiciones políticas y económicas concretas ${ }^{5}$. Barnes argumenta, por ejemplo, que la expansión de la economía de mercado libre a nivel mundial ha producido una exclusión sistemática de las personas discapacitadas, relacionando directamente factores políticos y económicos macrosociales con la exclusión de las $\mathrm{PD}^{24}$.

\section{Colaboradores}

U Díaz-Aristizabal, S Sanz-Victoria, M Sahonero-Daza, S Ledesma-Ocampo, M CachimuelVinueza y M Torrico participaron en igual de todas las etapas de elaboración del artículo.

\section{Agradecimientos}

En la elaboración de este trabajo hemos recibido la colaboración de Ángel Martínez Hernáez y Susan DiGiacomo, profesores del Departamento de Antropología de la Universitat Rovira i Virgili (Tarragona), a los que agradecemos sus inestimables aportaciones. Agradecemos además la cariñosa acogida que nos concedieron los integrantes de la Fundación Arquidiocesana Padre Ignacio Zalles a los extranjeros que colaboramos temporalmente en la fundación. 


\section{Referencías}

1. World Health Organization (WHO). Disability and Rehabilitation Team (DAR). World Report on Disability and Rehabilitation. [consultado 20 Nov 2011]. Disponible en: http://www.who.int/disabilities/ publications/dar_world_report_concept_note.pdf

2. Krefting D. Understanding Community Approaches to Handicap in Development (CAHD). Handicap International. 2001. [consultado 20 nov 2011]. Disponible en: http://digitalcommons.ilr.cornell.edu/cgi/ viewcontent.cgi article $=1434 \&$ context $=$ gladnetcollect

3. United Nations (UN). Factsheet on persons with disabilities. United Nations (UN). Nueva York. Enable 2008. [consultado 20 nov 2011]. Disponible en: http:/ /www.un.org/disabilities/documents/toolaction/ pwdfs.pdf

4. Groce NE, Gannotti M. Marginados de la sociedad: los discapacitados de América Latina. En: The Internacional Development Research Centre (IDRC), editor. Derechos económicos, sociales y culturales en América Latina: del invento a la herramienta. México: Plaza y Valdéz SA; 2006. p. 361-382.

5. Menéndez EL. La parte negada de la cultura. Relativismo, diferencias y racismo. Barcelona: Edicions Bellaterra; 2002.

6. Elwan A. Poverty and disability: a survey of the literature. Washington: The World Bank; 1999.

7. Thomas P. Disability, poverty and the millennium development goals. New York: Cornell University; 2005.

8. Ramírez Hita S. Donde el viento llega cansado. Sistemas y prácticas de salud en la ciudad de Potosí. La Paz: Cooperazione Italiana; 2005.

9. Ramírez Hita S. Calidad de atención en salud. Prácticas y representaciones sociales en las poblaciones quechua y aymará del altiplano boliviano. La Paz: Organización Mundial de la Salud (OMS, OPS); 2009.

10. Tapia I, Royder R, Cruz T. ;Mentisan, paracetamol o wira wira? Jóvenes, salud e interculturalidad en los barrios mineros de Potosí. La Paz: Fundación PIEB; 2006

11. Helander E. Prejudice and dignity. An introduction to Community-Based Rehabilitation. New York: United Nations Development Programme; 1993.

12. World Health Organization (WHO). Declaration of Alma Ata. 1978. [consultado 20 nov 2011]. Disponible en: http://www.who.int/hpr/NPH/docs/declaration_ almaata.pdf

13. Helander E, Mandis P, Nelson G, Goerdt A. Training in the communitiy for people with disabilities. Ginebra: World Health Organization (WHO); 1989.

14. International Labour Organization (ILO), United Nations Educational, Scientific and Cultural Organization (UNESCO), World Health Organization (WHO). Community-Based Rehabilitation for and with people with disabilities. Ginebra: International Labour Organization (ILO), United Nations Educational, Scientific and Cultural Organization (UNESCO), World Health Organization (WHO); 1994.
15. International Labour Organization (ILO), United Nations Educational, Scientific and Cultural Organization (UNESCO), World Health Organization (WHO). CBR. A strategy for rehabilitation, equalization of opportunities, poverty reduction and social inclusion of people with disabilities. Ginebra: International Labour Organization (ILO), United Nations Educational, Scientific and Cultural Organization (UNESCO), World Health Organization (WHO); 2004.

16. Kronenberg F, World Federation of Occupational Therapists (WFOT-CBR Project Team). Position paper on CBR for the International Consultation on Reviewing CBR. Helsinki: World Health Organization (WHO); 2003.

17. García Recio E, Sanz Victoria S. Una introducción al concepto de RBC. Revista de la APETO 2005; 36(Especial Dependencia):23-30.

18. Organización Mundial de la Salud (OMS). Censo de personas con discapacidad año 2000 zona Alto Lima. La Paz: Organización Mundial de la Salud (OMS); 2000 .

19. Internatonal Disability Rights Monitor (IDRM). Regional Report of the Americas. 2004. [consultado 20 nov 2011]. Disponible en: http://www.idrmnet. org/pdfs/IDRM_Americas_Sp_04.pdf

20. Bolivia. Congreso Nacional. Ley No 1678 de la Persona con Discapacidad. La Paz: 1995; 15 dic.

21. Bolivia. Secretaría Nacional de Salud. Política Nacional de Prevención de Discapacidades y Rehabilitación en Salud. La Paz: Secretaría Nacional de Salud; 1996.

22. Aguirre Beltrán G. Programas de salud en la situación intercultural. México: Fondo de Cultura Económica; 1994.

23. Campos Navarro R. La Antropología médica en México. México: Universidad Autónoma Metropolitana; 1992.

24. Barnes C. Discapacidad, política y pobreza en el contexto del "Mundo Mayoritari". Política y Sociedad 2010; 47(1):11-25.

Artigo apresentado em 25/10/2009

Aprovado em 21/02/2010

Versão final apresentada em 15/03/2010 